\title{
Modelagem numérica da formação do Rifte Continental do Sudeste do Brasil
}

Rafael Monteiro* (IAG/USP), Victor Sacek (IAG/USP)

Copyright 2016, SBGf - Sociedade Brasileira de Geofísica

Este texto foi preparado para a apresentação no VII Simpósio Brasileiro de Geofísica, Ouro Preto, 25 a 27 de outubro de 2016. Seu conteúdo foi revisado pelo Comitê Técnico do VII SimBGf, mas não necessariamente representa a opinião da SBGf ou de seus associados. É proibida a reprodução total ou parcial deste material para propósitos comerciais sem prévia autorização da SBGf.

\section{Abstract}

The Continental Rift of Southeastern Brazil is a tectonic feature which expressively influenced the sediment transport pattern in eastern Brazilian divergent margin. The genesis of this rift is quantitatively analyzed taking into account how different stresses sources may have influenced its formation. Using a 2D viscoelastic numerical model we analyse the lithospheric state of stresses due the effects of erosion and sedimentation and show that a large area of the lithosphere are affected by this loading/unloading variation in the topography. The loading due to sedimentation also contribute to increase the non-lithostatic stresses under the area being eroded. Quantitative constrain to geological models of the evolution of southeastern Brazilian continental margin may be obtained which are important for offshore oil exploration analysis.

\section{Introdução}

O Rifte Continental do Sudeste do Brasil (RCSB) (Riccomini et al., 2004) é uma feição tectônica que se estende por $c$. $1000 \mathrm{~km}$ paralelamente à costa, na região emersa do continente, sendo relacionado à formação de diversas bacias cenozóicas. As feições morfoestruturais na parte central do RCSB são a planície costeira, a Serra do Mar, o vale do Rio Paraíba do Sul, e a Serra da Mantiqueira (Hiruma et al., 2010) (Figura 1). Nessa porção também ocorre a maior bacia do RCSB, a Bacia de Taubaté, cujo pacote sedimentar atinge até $800 \mathrm{~m}$ de espessura (Riccomini et al., 2004).

Estudos de cronoestratigrafia nas Bacias de Santos e Campos indicam que ocorreu uma contínua diminuição de fornecimento de sedimentos, no final do Cretáceo e início do Terciário, na Bacia de Santos, e aumento na sedimentação clástica na região a nordeste desta, na Bacia de Campos (Karner \& Driscoll, 1999). Essa mudança de padrão é interpretada como consequência da formação do RCSB, resultando na captura de drenagem do interior do continente para o Rio Paraíba do Sul, passando a transportar sedimentos para a Bacia de Campos e consequentemente diminuindo a sedimentação na Bacia de Santos. Esse processo mostra a importância do entendimento da formação do rifte continental e sua influência na evolução geológica da margem continental no sudeste do Brasil, sendo um fator importante também para as análises de risco exploratório na indústria de petróleo.
Diversos modelos foram propostos para a formação do RCSB, porém, ainda existe debate sobre o tema (Riccomini, 1989; Zalán \& Oliveira, 2005; Cobbold et al., 2005; Cogné et al., 2011). Por exemplo, Riccomini (1989) concluiu que a formação do RCSB e o preenchimento sedimentar e vulcânico das bacias formadas ocorram no Paleógeno, sob a ação de esforços distensivos, que resultaram na reativação de antigas zonas de cisalhamento como falhas normais. Outros modelos envolvem o soerguimento da região, devido a uma anomalia térmica, cujo posterior colapso resultou na formação dos grábens que abrigam as bacias cenozóicas (e.g. Zalán \& Oliveira, 2005).

Embora diversos modelos tratem da origem do RCSB, nenhum trabalho analisa através de experimentos numéricos se os mecanismos propostos são fisicamente viáveis para a gênese do RCSB.

Neste trabalho, o efeito da erosão e sedimentação é analisado a partir de um modelo numérico que simula o comportamento reológico da litosfera ao longo do tempo, avaliando quantitativamente como esses processos geológicos afetam os esforços na litosfera.

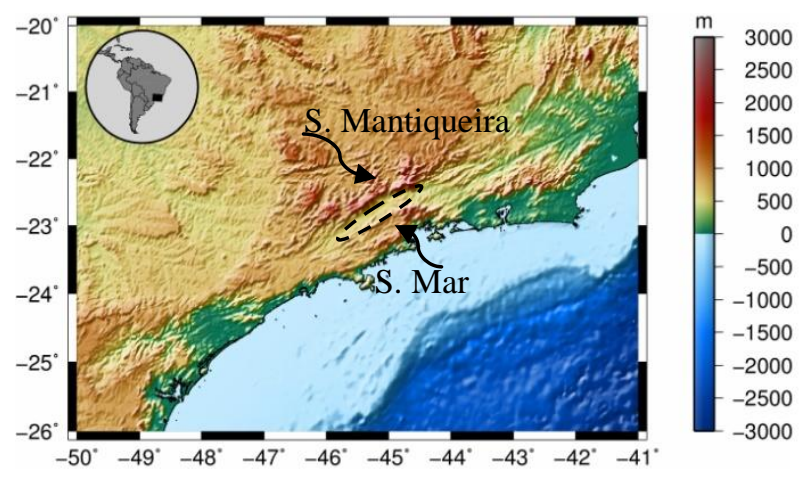

Figura 1 - Topografia e região central do RCSB. A topografia é marcada pelas escarpas das Serras do Mar e Mantiqueira (indicadas pelas setas). A maior bacia do RCSB ocorre entre essas serras (contorno tracejado).

\section{Modelagem dos esforços}

A resposta da litosfera aos esforços depende da escala de tempo no qual esses esforços atuam. Neste trabalho, o comportamento reológico da listosfera é modelado como um material viscoelástico, representativo dos efeitos dos esforços sobre a litosfera ao longo de dezenas de milhares de anos.

As equações de equilíbrio de esforços são dadas por

$$
\sigma_{i j, j}+f_{i}=0 \quad \text { em } \Omega
$$




$$
\begin{array}{cc}
u_{i}=q_{i} & \text { em } \Gamma_{q_{i}} \\
\sigma_{i j} n_{j}=h_{i} & \text { em } \Gamma_{h_{i}}
\end{array}
$$

em que $\sigma_{i j}$ são as componentes do tensor de esforços, $f_{i}$ as forças de corpo, $\Omega$ é o domínio com contorno $\Gamma_{q_{i}}$ e $\Gamma_{h_{i}}$ nos quais o deslocamento é especificado por $q_{i}$ e as trações por $h_{i}$, respectivamente. O comportamento reológico da listosfera é determinado pelo modelo de Maxwell de um material viscoelástico, no qual a deformação é distribuída entre as componentes elástica $\left(\varepsilon^{e}\right)$ e dúctil $\left(\boldsymbol{\varepsilon}^{v}\right)$ :

$$
\begin{gathered}
\boldsymbol{\sigma}=\boldsymbol{\sigma}^{e}=\boldsymbol{\sigma}^{v} \\
\boldsymbol{\varepsilon}=\boldsymbol{\varepsilon}^{e}+\boldsymbol{\varepsilon}^{v}
\end{gathered}
$$

Os esforços são calculados pela equação

$$
\begin{gathered}
\dot{\boldsymbol{\sigma}}=\boldsymbol{D}\left(\dot{\boldsymbol{\varepsilon}}-\dot{\boldsymbol{\varepsilon}}^{v p}\right) \\
\boldsymbol{D}=\frac{E}{(1+v)(1-2 v)}\left[\begin{array}{ccc}
(1-v) & v & 0 \\
v & (1-v) & 0 \\
0 & 0 & (1-2 v)
\end{array}\right] \\
\dot{\boldsymbol{\varepsilon}}^{v p}=\frac{\sigma^{n-1}}{4 \eta}\left[\begin{array}{ccc}
1 & -1 & 0 \\
-1 & 1 & 0 \\
0 & 0 & 2
\end{array}\right]\left[\begin{array}{c}
\sigma_{x x} \\
\sigma_{y y} \\
\sigma_{x y}
\end{array}\right]
\end{gathered}
$$

em que $E$ é o módulo de elasticidade, $v$ é o coeficiente de Poisson, $\eta$ é a viscosidade, $n$ é o expoente da lei de potência de um fluido não linear e $\sigma$ é dado por

$$
\sigma=\sqrt{\left(\frac{\sigma_{x x}-\sigma_{y y}}{2}\right)^{2}+\sigma_{x y}^{2}}
$$

As equações são resolvidas pelo método dos elementos finitos adotando um algoritmo explicito na discretização das derivadas temporais (Melosh \& Raefsky, 1980). O modelo numérico deste trabalho é adaptado de Assumpção \& Sacek (2013).

\section{Validação do modelo numérico}

A validade do modelo numérico foi verificada comparando-se a solução analítica de um problema 1D apresentado por Kusznir e Bott (1977) com o resultado numérico do modelo. A solução analítica fornece o esforço horizontal ao longo do tempo em um modelo simplificado de litosfera composto por duas camadas viscoelásticas de mesmo módulo de elasticidade $(E)$ : uma superior, de espessura $l_{s}$ e viscosidade $\eta_{s}$, e uma inferior, de espessura $l_{i}$ e viscosidade $\eta_{i}$. Considerando os valores $E=70 \mathrm{GPa}, \eta_{s}=10^{24} \mathrm{~Pa} \cdot \mathrm{s}$ e $\eta_{i}=10^{23} \mathrm{~Pa} \cdot \mathrm{s}$, o resultado numérico apresentou uma diferença máxima menor do que $2 \%$ apenas no intervalo transiente da resposta viscoelástica do modelo, no qual os esforços variam ao longo do tempo (Figura 2). Para o intervalo de tempo posterior, a solução numérica convergiu para a solução analítica.

\section{Resultados}

Para avaliar o efeito da variação da carga topográfica e sedimentar no estado de esforços da litosfera foram simulados dois cenários denominados $\mathrm{A}$ e $\mathrm{B}$. No cenário $A$, foi simulado a erosão de uma carga topográfica, existente desde o início da simulação, com uma taxa de erosão linear por toda sua extensão, enquanto no cenário $B$, além da erosão da área continental (igual ao modelo A), foi adicionado um carregamento, com uma taxa linear, na região correspondente à área submersa do modelo, simulando o preenchimento sedimentar de uma bacia marginal (Figura 3). Considerou-se uma carga topográfica (cenário A) de $3 \mathrm{~km}$ de elevação com extensão de $240 \mathrm{~km}$ (Figura 3, setas vermelhas) e, no cenário B uma carga equivalente foi adicionada como carregamento (Figura 3, setas azuis). O período de variação das cargas, em ambos os cenários, foi de 30 milhões de anos (Myr). O centro do modelo (em $800 \mathrm{~km}$ ) corresponde à transição entre a área emersa e submersa.

Os esforços principais desconsiderando a componente litostática, isto é, os esforços não litostáticos, são apresentados na Figura 4 para ambos os cenários no instante de tempo de 30 Myr após o início da simulação, e correspondente ao fim do intervalo de variação das cargas sobre o modelo.

O efeito observado da erosão (cenário A, Figura 4A) no padrão de esforços não litostático na litosfera é caracterizado por distensão na parte superior da crosta e compressão na parte superior do manto litosférico. $\mathrm{Na}$ crosta, a magnitude dos esforços diminui com a profundidade até se tornarem compressivos acima da crosta inferior. Nas regiões adjacentes à área onde ocorre erosão o padrão de esforços é compressivo e a magnitude decai tanto em profundidade como lateralmente, com aumento da distância à área erodida. No cenário $B$, onde, além da erosão, exsite 0 carregamento correspondente à sedimentação, o padrão geral de esforços sob a área erodida foi mantido, com diferença na magnitude dos esforços que se tornaram maiores, na região adjacente ao carregamento, em relação ao cenário $A$. Já na região do carregamento, o padrão de esforços é essencialmente oposto àquele da área erodida e magnitude ligeiramente inferior.

$\mathrm{Na}$ Figura 4, também nota-se que entre a crosta superior e média e a parte superior do manto litosférico, existe uma zona em que não há acúmulo de esforços e que corresponde à base da crosta. Desse modo, nessa localização, a viscosidade efetiva é baixa o suficiente para atenuar os esforços e não há concentração de tensão ou compressão.

\section{Discussão e Conclusões}

Os cenários numéricos de erosão e carregamento sedimentar analisados neste trabalho mostram que a litosfera é afetada por variações de carga na superfície desde a superfície até a porção superior do manto litosférico. Gallagher et al. (1994) sugeriram que houve a denudação de ao menos $3 \mathrm{~km}$ na região costal e de $1 \mathrm{~km}$ no interior do continente após a ruptura continental e abertura do oceano Atlântico. Desse modo, o efeito causado pela erosão e sedimentação pode ter 
influenciado significativamente o padrão de esforços na litosfera na região sudeste do Brasil, e assim, ter sido responsável pela reativação das zonas de fraquezas que deram origem ao RCSB.

Este trabalho será aprimorado e as próximas etapas irão avaliar através da análise de critérios de ruptura, como por exemplo, o critério de Byerlee, as regiões da litosfera que são susceptíveis à ruptura e como elas são afetadas por diferentes processos geológicos. Adicionalmente será avaliado como os esforços intraplaca afetam o estado de esforços da litosfera em conjunto com as variações de carga.

\section{Agradecimentos}

À ANP e à PETROBRÁS pelo apoio financeiro concedido à R. Silva (bolsa de Mestrado) através do Programa de Recursos Humanos da ANP (PRH 19).

\section{Referências}

ASSUMPÇÃO M \& SACEK V. 2013. Intra-plate seismicity and flexural stresses in central Brazil. Geophysical Research Letters, 40 (3), 487-491.

COBBOLD PR, MEISLING KE \& MOUNT VS. 2001. Reactivation of an obliquely rifted margin, Campos and Santos basins, southeastern Brazil. AAPG bulletin, 85 (11), 1925-1944.

COGNÉ N, GALLAGHER K \& COBBOLD PR. 2011. Post-rift reactivation of the onshore margin of southeast Brazil: Evidence from apatite (U-Th)/He and fission-track data. Earth and Planetary Science Letters, 309 (1), 118130.

GALLAGHER K, HAWKESWORHTH CJ \& MANTOVANI MSM. 1994. The denudation history of the onshore continental margin of SE Brazil inferred from apatite fission track data. Journal of Geophysical Research: Solid Earth, 99 (B9), 18117-18145.

HIRUMA ST, RICCOMINI C, MODENSEI-GAUTTIERI $M C$, HACKSPACHER PC, NETO JCH, \& FRANCOMAGALHAES AO. 2010. Denudation history of the Bocaina Plateau, Serra do Mar, southeastern Brazil: Relationships to Gondwana breakup and passive margin development. Gondwana Research, 18 (4), 674-687

KARNER GD \& DRISCOLL NW. 1999. Tectonic and stratigraphic development of the West African and eastern Brazilian Margins: insights from quantitative basin modelling. Geological Society, London, Special Publications, 153, 11-40.

KUSZNIR NJ \& BOTT MHP. 1977. Stress concentration in the upper lithosphere caused by underlying viscoelastic creep. Tectonophysics, 43 (3-4), 247-256.

MELOSH HJ \& RAEFSKY A. 1980. The dynamical origin of subduction zone topography. Geophysical Journal International, 60 (3), 333-354.
RICCOMINI C, SANT'ANNA LG \& FERRARI AL. 2004. Evolução geológica do rift continental do Sudeste do Brasil. In: MANTESSO NETO V, BARTORELLI A, CARNEIRO CDR \& NEVES BBB (eds), Geologia do continente Sul-Americano: evolução da obra de Fernando Flávio Marques de Almeida, Editora Beca, São Paulo, pp. 383-405.

ZALÁN PV \& OLIVEIRA J. 2005. Origem e evolução estrutural do Sistema de Riftes Cenozóicos do Sudeste do Brasil. Boletim de Geociências da Petrobrás, 13 (2), 269-300. 

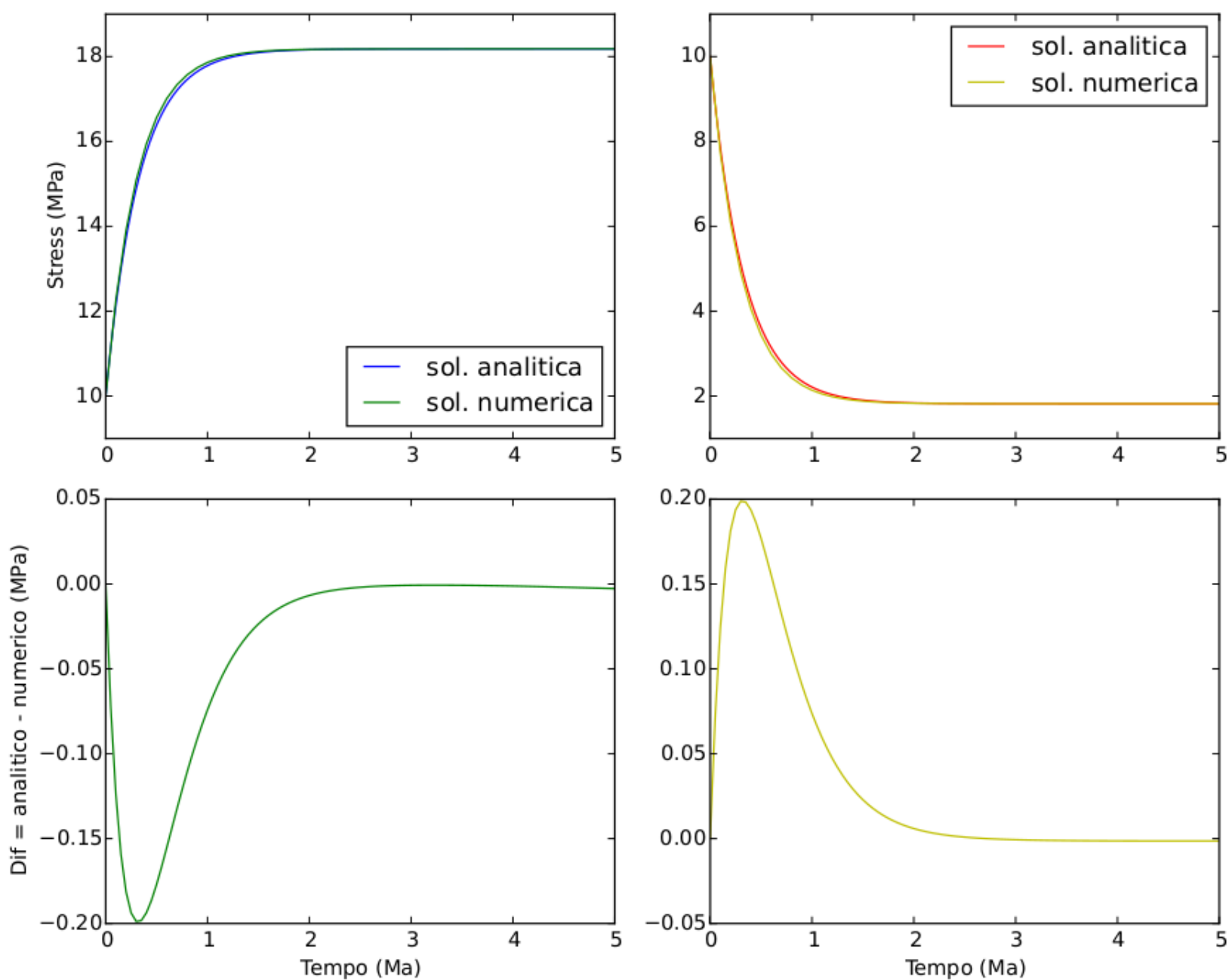

Figura 2 - Esforço horizontal e diferença entre a solução analítica e numérica na camada superior (esquerda) e inferior (direita) do modelo.

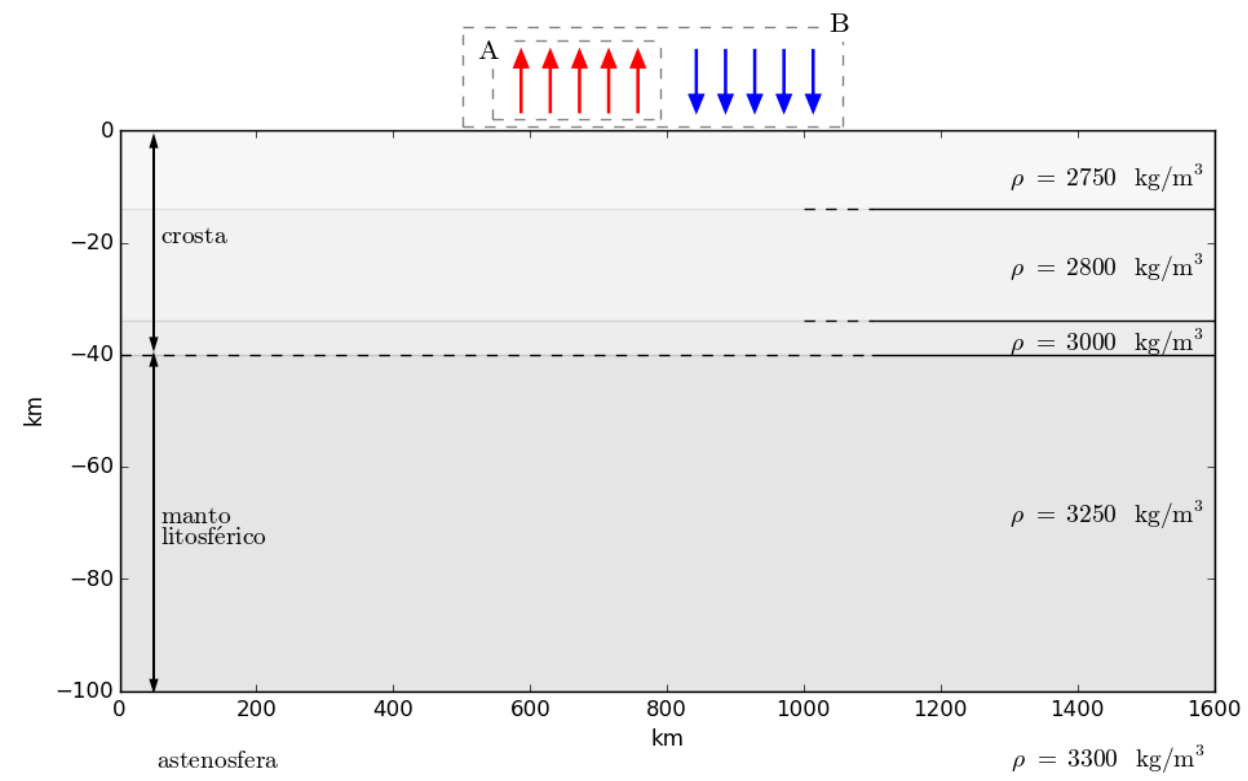

Figura 3 - Configuração do modelo numérico. As setas vermelhas e azuis indicam erosão e sedimentação, respectivamente, no modelo $A$ (apenas erosão) e $B$ (erosão e sedimentação). 

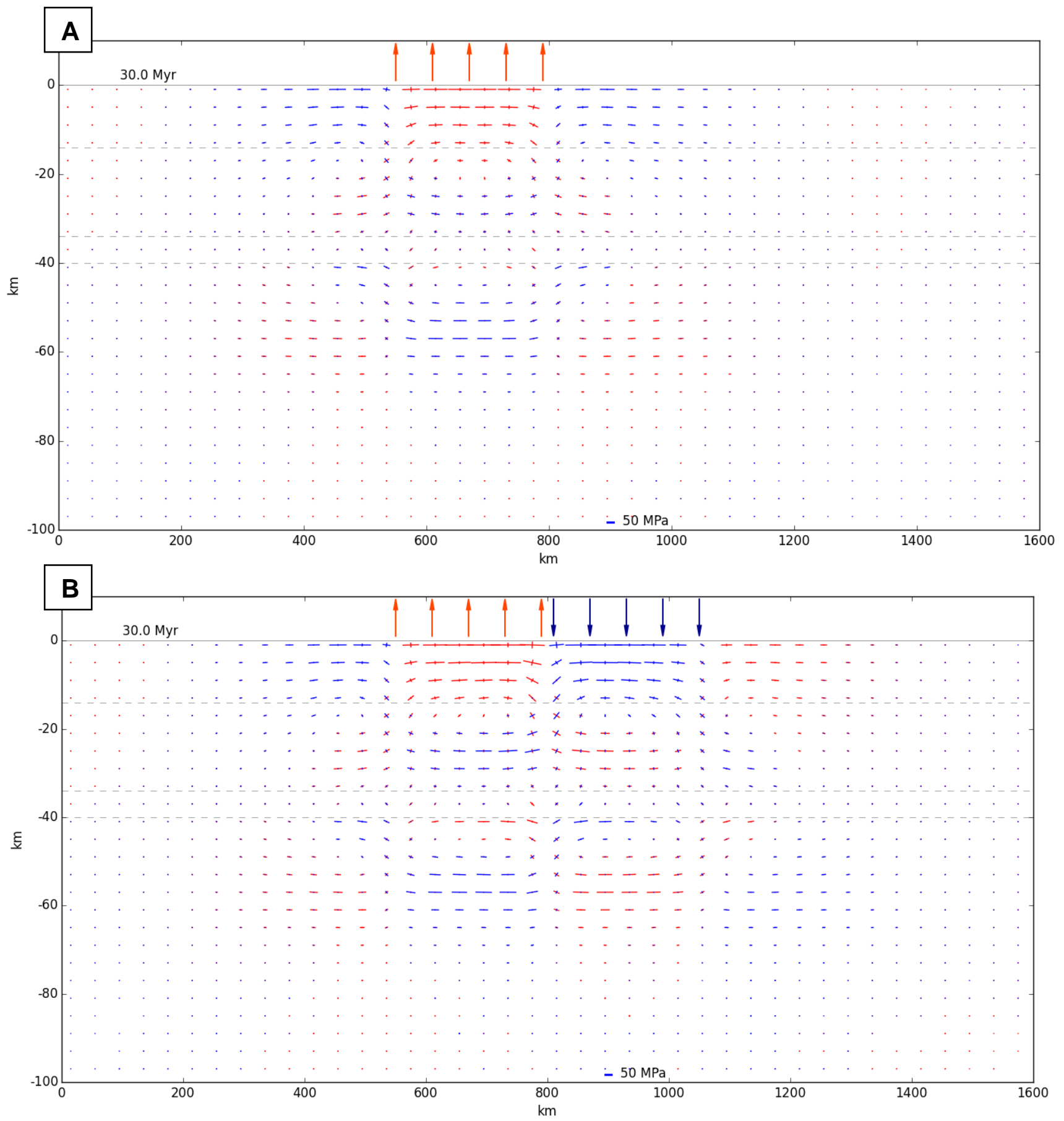

Figura 4 - Esforços não litostático na litosfera. Os traços vermelhos indicam distensão e os traços azuis indicam compressão. As setas vermelhas e azuis, na parte superior, indicam erosão e sedimentação, respectivamente, no modelo $A$ (apenas erosão) e $B$ (erosão e sedimentação) e as linhas tracejadas horizontais indicam as divisões na crosta e interface crosta-manto. 